\section{Cahiers balkaniques}

$36-37$ | 2008

L'image de la période ottomane dans les littératures balkaniques

\title{
De l'honorable ennemi au compagnon de fortune
}

From the respectable enemy to the one who shares a common destiny

\section{Györgyi Mate}

\section{CpenEdition}

\section{Journals}

Édition électronique

URL : https://journals.openedition.org/ceb/1531

DOI : $10.4000 /$ ceb. 1531

ISSN : 2261-4184

Éditeur

INALCO

\section{Édition imprimée}

Date de publication : 1 décembre 2008

Pagination : 213-230

ISBN : 978-2-85831-173-6

ISSN : 0290-7402

\section{Référence électronique}

Györgyi Mate, « De l'honorable ennemi au compagnon de fortune », Cahiers balkaniques [En ligne], 36-37 | 2008, mis en ligne le 16 mai 2012, consulté le 06 juillet 2021. URL : http:// journals.openedition.org/ceb/1531; DOI : https://doi.org/10.4000/ceb.1531

Ce document a été généré automatiquement le 6 juillet 2021.

Cahiers balkaniques est mis à disposition selon les termes de la Licence Creative Commons Attribution - Pas d'Utilisation Commerciale 4.0 International. 


\title{
De l'honorable ennemi au compagnon de fortune
}

\author{
From the respectable enemy to the one who shares a common destiny
}

\author{
Györgyi Mate
}

1 Dans la culture hongroise, 150 ans de proche cohabitation avec l'Empire ottoman ont laissé d'innombrables traces, depuis des trilogies romanesques jusqu'aux réminiscences poétiques à peine perceptibles. L'effet est analogue dans l'architecture : on trouve dans la Hongrie actuelle une mosquée dite la plus vaste d'Europe Centrale, mais aussi des signes effacés dans des briques du seizième siècle. La confluence est telle que, par exemple, certaines fioritures dans la musique populaire hongroise ne sont plus reconnues comme emprunts. La richesse relative en documents ne nous permet évidemment que de frôler la «question turque». Dans le cadre d'une courte contribution, au lieu d'esquisser un tableau général de l'opinion publique hongroise sur les Ottomans ou d'en examiner systématiquement le développement, je me propose de me pencher ici sur un seul élément des clichés dont l'empreinte est visible dans la littérature hongroise. La question qui surgit dans presque tous les écrits est celle de l'honneur et tout particulièrement de la parole donnée. Trois aspects de la problématique s'offrent naturellement à l'analyse. Les engagements personnels politiques et militaires - sont saisis par les contemporains comme des affaires morales. Le problème des conversions, également abondamment documenté, s'aligne sur le domaine de la religion. Il est plus difficile de suivre le fonctionnement de la cohabitation. Le règlement des menues affaires quotidiennes, ce qu'on ne peut saisir qu'à travers la mémoire des cas de justice, pourra être traité à l'intérieur du champ politique triangulaire (à savoir, les puissances rivales de l'Occident, y compris la Hongrie intégrée à l'Empire Habsbourg et l'Empire ottoman). Les genres des textes à l'appui de ces trois approches sont d'une grande diversité, le mémoire d'esclave ou le chant historique y tiennent une place égale avec le roman du XIX ${ }^{e}$ siècle.

2 Au temps de la première apparition des Ottomans, les notions "Ismaélite », "Turcs infidèles », « Sarrasins », empruntés aux guerriers et pèlerins rentrés de la Terre sainte, désignaient, assez confusément, les peuples musulmans de l'Asie mineure ${ }^{1}$. Après les 
rapports militaires qui ne parlaient que d'attaques-surprises, menées avec un courage fanatique, les premières sources nuancées sont les écrits des esclaves qui pouvaient rentrer dans leur pays après avoir passé de longues années dans l'Empire ottoman. ${ }^{2}$

Contrairement aux avis négatifs des auteurs ultérieurs et occidentaux, le dominicain transylvain Georgius de Hungaria ${ }^{3}$ présente non pas un tyran sanguinaire, mais un sultan très simple et pieux, juste et proche de ses sujets. L'État basé sur les valeurs militaires réunit de vaillants guerriers disciplinés. La simplicité, la modération, la bienveillance sont des vertus collectives, le comportement des femmes est exemplaire. Les Turcs sont modestes dans leurs habitudes vestimentaires, parcimonieux dans la consommation, et bénévoles dans le don et dans la guerre. Les voyageurs diplomates relatent plus tard parfois avec incompréhension leur sens de la propreté dont frère Georges loue l'utilité.

4 Ce futur dominicain est le premier à se familiariser plus profondément avec la doctrine islamique. Il va jusqu'à prêcher aux musulmans et à les conseiller dans certaines questions de foi. Son mémoire décrit les ordres religieux, notamment les derviches, et résume les vertus des grands saints musulmans. La tolérance et l'intérêt envers les formes de piété sont illustrés par une histoire liée aux religieux de Péra. Après avoir présenté leur église et leur liturgie au sultan, celui-ci a permis, à ses frais, l'institution d'un évêque pour la consolation des brebis abandonnées.

$5 \quad$ L'image du plus grand seigneur et des petites gens est également positive sous la plume de Georgius de Hungaria. Il a vécu, dès le premier moment de sa captivité, la fiabilité de la parole des musulmans. Le sultan Murat II, chef de l'armée ottomane a promis aux bourgeois de Sebes de pouvoir se retirer librement sous la tutelle du voïvode Vlach Dracul ; le jeune Georgius les a vus quitter les lieux de ses propres yeux, à partir de la tour dans laquelle il s'était isolé avec d'autres résistants. La simplicité et le comportement pondéré des Ottomans viennent de l'intérieur, écrit-il dans le $15^{\mathrm{e}}$ chapitre de son mémoire, leur honnêteté prend sa source dans un penchant naturel, un sentiment propre et vertueux. Ainsi, leurs habitudes peuvent être erronées et considérées comme incorrectes, mais méritent tout de même plus l'acquittement que les manières polies dont l'origine n'est que corporelle, extérieure et par conséquent vicieuse. Cette constatation - selon l'opinion de son éditeur en hongrois, Lajos Tardy s'est formée sur place, dans l'esprit d'un témoin direct, et non pas dans le cerveau d'un dominicain âgé qui a probablement dû céder à l'exigence d'un compromis, car, dans la deuxième partie de l'œuvre, il réfutera et retournera trait par trait les éléments positifs de la première partie. Il n'en reste pas moins que dans son intégrité, l'œuvre de Georgius de Hungaria est une ouverture dans une période où, les peuples des Balkans et la Hongrie exceptés, l'Europe n'avait pas encore une expérience de première main sur l'Empire ottoman.

Un siècle après, ce même empire prend un tout autre aspect sous la plume d'un curieux voyageur, Hans Dernschwam ${ }^{4}$. En 1548, les Fugger se sont pratiquement retirés des affaires de la Haute-Hongrie. Dernschwam prend sa retraite et se consacre à ses livres dans son manoir de Cseszte, ou rend visite à ses amis humanistes à Vienne. En 1553, il prend l'initiative d'accompagner, à ses propres frais, individuellement, l'ambassade impériale-royale à Istanbul. Les raisons de sa requête adressée à la cour de Ferdinand Habsbourg ne sont pas connues. L'on peut évidemment supposer que sa soif humaniste de connaître le monde, le goût de l'aventure qui a poussé Guillaume Postel jusqu'à Constantinople et Sir Philip Sidney jusqu'en Hongrie, lui ont conseillé de profiter de 
l'occasion. Ou au contraire, on peut penser que ses anciens patrons devenus amis, les Fugger, lui ont demandé des informations directes sur l'Empire avec lequel ils ont déjà tenté d'avoir des contacts commerciaux vingt ans auparavant. Qu'une paix durable avec le Grand Turc aurait permis l'exploitation minière en territoire ottoman, cette pensée avait déjà germé dans la tête de cet important homme d'affaires de la Renaissance. Antoine Fugger a marié dans ses projets un réalisme économique minutieux et l'élan hardi d'un explorateur. Le fait que le manuscrit de la relation se trouvait dans les archives des Fugger, ne prouve en rien cette hypothèse suggérée par Lajos Tardy, mais les opinions très libérales, voire antiimpériales et antiecclésiastiques de Dernschwam, son point de vue ramené essentiellement au matériel et aux faits positifs révèle en lui un mémorialiste qui regarde les conditions de l'Empire ottoman d'un œil critique.

7 L'Itinéraire de Turquie est né entre 1553 et 1555, à l'apogée de l'Empire, mais montre déjà, surtout dans le miroir du mémoire de Georgius de Hungaria, les signes de la décadence. Le sultan est devenu le Grand Seigneur inapprochable, au lieu des féaux guerriers puritains, l'auteur trouve des soldats que seuls l'odeur de la proie et l'espoir de l'enrichissement motivent, qui ravagent partout, malgré leur solde et ravitaillement réguliers. De plus, les "vrais Turcs» (dénomination des Ottomans donnée par les auteurs hongrois de l'époque) n'ont plus le goût de la gloire, ils s'emparent plus aisément du pouvoir en usant des moyens de ruse et de violence. La guerre est devenue l'affaire des janissaires ${ }^{5}$. Les soldats se sont réduits à des seigneurs efféminés et la cruauté est devenue gratuite et raffinée.

L'ambiance de confiance et de communauté s'est déformée en un état de violence étatique et d'espionnage du voisin. La consommation de vin et d'opium, devenue quotidienne, était un fait largement connu et relaté en Hongrie ${ }^{6}$. La discipline, le sens de la vertu et des valeurs - comme la simplicité - constatés par le frère Georgius ont disparu non seulement de la guerre, mais de la vie quotidienne également. À la fidélité maritale, s'est substituée une vie de débauche des hommes et des femmes, les paysans n'ont plus envie de travailler les terres, et le manque de liberté dans le commerce et les exploitations diverses empêche le développement de l'industrie et des échanges. La pratique de la religion n'est qu'extérieure, et la piété même des prêtres s'est muée en esclavagisme: Dernshwam ne leur reconnaît que le zèle tourné en fanatisme. La religiosité des gens a diminué ; il est interdit de parler des questions de foi. Toutefois, les conversions sont encouragées et même forcées. En affaires de justice, les témoignages s'achètent pour quelques pièces d'argent. Dernschwam raconte, entre autres, l'histoire d'un armurier captif, Hans Schaczel, qu'il a racheté de son maître musulman. Le tchaüs chargé d'accompagner les ambassadeurs hongrois, apercevant le changement de vêtement de l'armurier qui voulait se joindre à la suite de Dernschwam, l'a retenu pendant une nuit, et

«voulait le convertir en Turc par la force. Comme Schaczel, en lui répliquant, s'y est opposé, il a dû supporter les gifles de celui-là, car si un chrétien - et qui plus est, un esclave - frappe un büssürmen, il sera non seulement battu, mais peut se retrouver au bûcher. $»^{7}$

Cette remarque de Dernschwam, notée certainement dans le feu des événements auxquels il a assisté, montre son parti pris et prouve son aveuglement, car malgré toutes sortes de tortures, si cruelles soient-elles, le bûcher n'a jamais figuré parmi les sanctions ottomanes. Dernschwam raconte donc quelques scènes non exemptes d'erreurs qui sont à prendre avec précaution. Toutefois, nous n'avons pas à douter du fait que l'on ait examiné publiquement si l'esclave était circoncis ou pas, que son sauf- 
conduit attestant sa libération, obtenu du kadi, n'ait servi à rien, et que Dernschwam ait dû payer une deuxième fois la rançon de l'armurier Schaczel. Tout s'achète en Turquie, mais aucun présent ni aucune somme payée ne sont des investissements sûrs, conclut l'ancien représentant des Fugger. Le déplacement dépend également des droits illégaux de passage ${ }^{8}$. Les envoyés, personnages importants, étaient accompagnés non seulement de spahis, mais aussi d'un tchaüs responsable de leur sécurité et bien-être, cette protection assurée par le pouvoir central de la Porte se transforme souvent en mise en demeure surveillée.

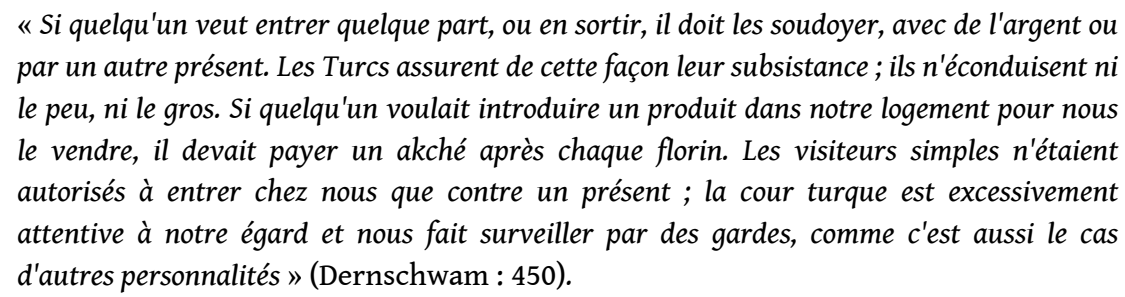

10 L'image noire peinte par Dernschwam, ce représentant de la haute bourgeoisie, cosmopolite et érudit, se confirme partiellement dans les écrits des auteurs qui ne sont jamais entrés à l'intérieur de l'Empire ottoman, mais avaient en revanche l'expérience de ses confins en Europe centrale. Les mémoires de Georgius de Hungaria et de Hans Dernschwam sont généralement accessibles, puisqu'écrits respectivement en latin et en allemand. Les chants du dernier jongleur, Sebastian Tinódi dit le Ménestrel sont, quant à eux, révélateurs d'une opinion publique qu'ils ont d'ailleurs formée et orientée.

11 Tinódi, d'humble origine, est devenu familier (serf curial) du seigneur Bálint Török, aristocrate dominant le Sud-Ouest de la Hongrie. Le jeune serf talentueux, comme le laisse deviner son érudition musicale et humaniste manifeste dans ses œuvres, a dû suivre des études avancées, sans toutefois les parfaire dans une université étrangère. En Hongrie, le genre du chant historique prend sa forme définitive sous sa plume ; entre 1538 et 1556, tous les événements importants de son époque lui servent de sujet d'inspiration. Son recueil intitulé Cronica compte toujours pour une source précieuse d'un témoin direct. Ses thèmes et ses explications sont exploités par toutes les tendances d'interprétation historique et littéraire ultérieures. Les Protestants puisent dans ses lamentations, les humanistes et le naturalisme insistent sur les erreurs et les bavures politiques intérieures, le romantisme y trouve une source d'émotions et d'événements exceptionnels.

Dans le poème intitulé La captivité de Péter Prini, István Majlát et Bálint Terek, Tinódi explique clairement la logique musulmane face aux infidèles :

La foi du Turc tient ceci fermement :

S'il trompe les kaurs avec de beaux mots et la foi

S'il peut les prendre par force ou avec des présents

Dieu lui donne le Paradis pour cela.

Durant le calme relatif entre 1545 et 1552, Tinódi remonte d'une douzaine d'années dans l'histoire, pour relater le Combat du sultan Soliman avec le pacha Kazul (1546). Le récit présente la rivalité « des Turcs rouges " guidés par Sahit Ahmaze, pacha Kazul (c'est-à-dire, Tahmasp Ir de la dynastie des Safavides) et les "Turcs blancs ", à savoir, les Ottomans. Soliman récompense cruellement l'opium offert par l'ambassadeur du shah, pris comme un présent téméraire : il le fait pendre par les jambes. Ses pachas lui déconseillent ce traitement, car « ils n'ont jamais entendu ni lu, qu'un ambassadeur n'ait été tué nulle part» (Tinódi: XIV.60-64). Ce n'est donc pas l'obligation de 
l'hospitalité et l'honneur orientaux que Tinódi fait invoquer aux pachas, néanmoins le sultan cède à ses conseillers, visiblement cultivés à la façon occidentale. Le sultan vainqueur passe une seule nuit avec la belle femme du pacha Kazul, puis la donne en mariage à un de ses beys. La femme inconsolable tue ce nouvel époux forcé. La punition du meurtre sera généralement connue en Hongrie et en Europe : elle sera jetée à la mer. La riposte du pacha furieux est encore une leçon de tactique: les troupes peu nombreuses de Kazul consument lentement, mais gravement l'armée de Soliman par des escarmouches proposées par les conseillers au lieu d'une bataille ouverte ${ }^{10}$. Soliman, coincé, ordonne à son vizir Ibrahim de quitter la Valachie et de se joindre à lui. «Vaillant guerrier et fin stratège, Ibrahim attise la jalousie de Soliman, qui décide de l'assassiner, mais remet à plus tard l'exécution de son projet, en suivant en cela les conseils de son pacha Aias.» (v. 240-255). Le chant de Tinódi finit par des prophéties apocalyptiques sur les ruines de Babylon (Bagdad). Il évoque les hérésies, les errements des chrétiens comme causes du pouvoir indomptable des infidèles, dont la puissance immense est comparée, par ailleurs, à celle d'Alexandre. Le chant historique souligne la culture diplomatique et militaire des conseillers des deux partis qui rappellent notamment au sultan Soliman son intérêt et son devoir de tenir parole envers un ambassadeur.

Trois cents ans plus tard, la nouvelle de Mór Jókai tisse une histoire plus intime autour de ces mêmes personnes. Le parjure y est d'autant plus douloureux que Kazul et Soliman sont des ennemis tous les deux musulmans, tandis qu'Ibrahim, le protagoniste central de Jókai, est un serviteur privilégié du sultan. La nouvelle intitulée Le rêve de Soliman commence par la description lente d'un cortège qui suit le monarque à l'ouverture de la mosquée portant le nom de l'Empereur. Ce jour de joie et de gloires militaires, toutes les fissures causées par le temps dans l'ancienne Byzance s'effacent, le peuple est heureux de voir un jet d'eau et d'en goûter l'eau, tous les dignitaires reçoivent un avancement, et Ibrahim, le plus fidèle, devient gendre et fils adoptif du sultan. À cet instant, le récit reste un moment comme suspendu, le narrateur transmet la pensée unanime des gens de la cour: Ibrahim doit mourir. Cette pensée saugrenue doit effleurer aussi la fille, car elle exige de son père le serment que ne jamais dans sa vie, ordonner la mort de son époux. Une nette coupure de la nouvelle recommence l'histoire parmi les mêmes circonstances retournées à l'envers. Le cortège des flagellants annonce un jour de malheur. Accablé de nouvelles de défaites, Soliman jure que la personne qui lui apportera une mauvaise nouvelle sera exécutée. L'ouléma lui fait lire des lettres qui, à la fois, annoncent l'assassinat de son gouverneur de Hongrie, Gritti, et accusent Ibrahim de trahison. Le vieux sage assoupit la conscience du sultan furieux en lui citant le Coran : il peut décider la mort d'Ibrahim, car celui qui dort ne vit pas, alors, s'il le tue durant son sommeil, il ne manque pas à sa parole. Soliman laisse l'ouléma agir. Celui-ci s'introduit à trois reprises dans la chambre de nuit du sultan : en vain, il répond toujours, il veille. La deuxième nuit se passe de la même façon. À la fin de la troisième, Soliman voit sa fille courir près de lui, en l'accusant et lui demandant grâce pour son mari, trois fois de suite. Trois conceptions se succèdent dans les réponses rêvées: le refus ignorant la situation, d'abord, montre un sultan sûr de la loyauté de son bras droit, puis, il accorde sa grâce pour les deux cas, innocence ou culpabilité d'Ibrahim, ensuite, il veut sauver les amoureux en les envoyant en Palestine ; cela veut dire que sa pensée noircit, il se met à croire à la culpabilité. Les trois rêves reflètent la vérité de la fiction (la précipitation de la jeune femme, trois fois chassée du seuil de son père). Soliman, déchiré, est réveillé par l'ouléma qui apporte la 
tête d'Ibrahim. La structure tendue de la nouvelle relativement courte, l'alternance quasi régulière des descriptions et des dialogues, les répétitions parcimonieuses et le jeu des correspondances et des niveaux de la narration font un petit chef-d'œuvre de ce récit romantique de Jókai.

Pour revenir au modèle, certains chants de Tinódi, dont La chronique des empereurs turcs sont perdus, mais, entre autres, l'Histoire du pacha Ali de Buda et celle sur La chute de Buda et la captivité de Bálint Török sont des sources dans lesquelles s'esquissent assez clairement les contours d'une vision de l'honneur militaire et politique, généralement adoptée dans les cours seigneuriales et dans les riches bourgs hongrois. En 1541, après la mort du roi János Szapolyai, Bálint Török s'est engagé à soutenir le sultan Soliman contre Ferdinand de Habsbourg. Parmi les 200 vers de La chute de Buda, dans une douzaine se répète le mot foi, ou l'expression lettre de foi, toujours dans le sens de "promesse", d'" accord" ou "sauf-conduit». À la fin de l'introduction du chant (versets 25-39), Bálint Török, après avoir choisi «le camp de Soliman contre le prétendant Habsbourg", refuse de demander la parole du pasha Mehmed, car il pense qu'une telle demande susciterait un soupçon contre lui.

Si je demande une lettre de foi,

de ma loyauté on doutera,

je me confie donc à mon Dieu.

Beaucoup m'appellent "Turc aux cheveux ",

mais j'accepte, bons seigneurs, sachez bien,

de mal grél'affection des païens. 39-43.

15 Avant la prise de Buda par le sultan, Péter Perényi l'exhorte de nouveau à la précaution, en évoquant les exemples de ceux qui, malgré les sauf-conduits les plus engageants, ont été retenus et emprisonnés. Mais Bálint Török n'y pense pas. Par la suite, il est transféré très dignement à Istanbul sur le conseil des pachas qui le tiennent pour une menace du fait de sa vaillance et l'étendue de son pouvoir. La formulation de Tinódi ("ayant entendu ceci, le sultan consentit à le retenir », 145-146) suggère qu'il s'agit d'une décision politique, prise de mauvais gré, qui prévaut sur la parole donnée. En fait, le poète, sans dispenser le sultan de l'obligation de l'honneur, donne une explication plausible à la rétention de longue durée. La cause est en même temps flatteuse pour le prisonnier ${ }^{11}$.

Le pacha Ali Khadime, en revanche, est un chef militaire sage et prévoyant qui sait tenir parole. L'exposition du sujet de l'Histoire du pacha Ali de Buda insiste sur les qualités du protagoniste qui, chargé d'occuper les châteaux du Nord-Est hongrois, choisit les campagnes courtes, en évitant l'armée du " Diable $~^{12}$. Si, après s'être rendu, Mihály Vas finit captif malgré la promesse d'Ali, c'est parce que ses agresseurs l'accusent de parjure $^{13}$. L'odeur de la proie pousse à la traîtrise les soldats de Hollókő, dont le capitaine, par contre, peut se retirer librement sur la parole d'Ali ${ }^{14}$.Le siège du manoir de Drégely est le motif central du chant historique (v. 57-103). Le capitaine Szondi refuse la grâce d'Ali, et meurt en héros avec tous ses soldats. Avant le moment décisif, il envoie chez Ali ses deux jeunes pages chanteurs en l'exhortant à les prendre sous sa protection. Ali accepte la tutelle des jeunes nobles vêtus en écarlate et cède à la demande de Szondi : il fait chercher le corps de celui-ci, le réunit avec la tête jetée dans les fossés, et le fait enterrer en grande dignité.

Deux éléments du récit central, relativement long, seront repris dans la poésie épique ultérieure. Le premier motif est la préparation des héros au combat final en grande pompe. À la fin de l'épopée Le siège de Sziget, avant la confrontation finale, Zrínyi prend ses plus riches habits, glisse cent pièces d'or dans ses poches des deux côtés, met le feu 
au château intérieur. Szondi fait tuer les chevaux et brûle les réserves, mais comble ses pages chanteurs de toutes les richesses qu'ils peuvent porter.

Le deuxième sujet souvent exploité est le respect devant la mort courageuse : le vainqueur rend un dernier hommage solennel au vaincu, soit sur une demande expresse (comme dans le cas de Szondi) ou, sans avoir engagé sa parole, seulement inspiré par l'honneur.

19 La reprise la plus célèbre de l'histoire du siège de Drégely sera une ballade de János Arany, écrite en 1856. Arany, un des plus grands poètes de la littérature hongroise, adopte le point de vue d'un troisième barde (rôle emprunté dans d'autres ballades également) qui écoute la narration rétrospective des deux pages chanteurs. Ceux-ci, penchés sur un tertre marqué par une lance, se font mander par un messager d'Ali, qui les sollicite d'abord gentiment, en énumérant les fruits, les mets affriolants et la bienveillance mielleuse, puis passe aux menaces. La réponse est toujours une évocation des événements passés lors du siège. Au fur et à mesure que l'appel devient de plus en plus pressant, le récit devient plus intense, jusqu'au point culminant, où les deux monologues, jusque-là parallèles, quoique, thématiquement, se répondant avec un décalage dans le temps, se croisent et font unisson. Le messager, sortant de son rôle, reconnaît la précellence de l'ennemi mort : "Roustem fut-il seul, combattit encore, le genou rompu, le tendon cassé d'un boulet». Il rappelle en vain les pages à leur obligation de divertir le pacha Ali, en les menaçant d'emprisonnement et de bastonnade, le poème se clôt sur leur refus des propositions de l'assassin de leur ancien maître.

Le poème romantique d'Arany était entendu par les contemporains comme un chant patriotique visant non pas le pacha Ali, mais François-Joseph et les méthodes de la cour habsbourgeoise.

21 Le romantisme tourne ainsi le sujet ottoman soit en patriotisme où le despotisme turc est la couverture de la tyrannie viennoise, soit en réflexion abstraite ou nostalgique. Les racines de ces deux voies remontent aux deux positions hongroises de la deuxième moitié du XVI ${ }^{\mathrm{e}}$ siècle : la ferveur des intellectuels protestants et la sage résignation des humanistes. Les réformés, anciens élèves des universités allemandes et néerlandaises, pensaient que le Turc est un fléau de Dieu. La réflexion humaniste se modifie au cours des décennies de cohabitation tout en s'alimentant, d'ailleurs, aussi par le néostoïcisme très en vogue (dont les traductions de Juste Lipse témoignent). Les anciens élèves de Padoue, embauchés dans le service administratif des Habsbourg prônaient d'abord la centralisation et l'unification du pays sous la tutelle de la cour de Vienne. Les historiens Miklós Istvánffy et Ferenc Forgách reconnaissent vite que l'intérêt et la force des Habsbourg sont loin d'être suffisants pour libérer la Hongrie. Selon leur opinion, Ferdinand, sans argent, ne va jamais jusqu'au bout de ses projets, et au lieu de donner un soutien efficace aux chrétiens, ne fait que des promesses sans lendemain (Ruzsás : 331).

22 Les humanistes et les dirigeants transylvains jouent désormais le jeu de l'équilibre entre les puissances ${ }^{15}$. L'archiduché, pris entre deux feux, est sauvegardé par de «beaux mots, promesses et présents, et par tous les moyens possible » (332). Les érudits du tournant du siècle ont finalement opté pour une cohabitation paisible, ceci grâce à une politique d'équilibre pleine de ruse et de détours, héritée et, par la suite, exercée avec succès par la plupart des archiducs transylvains du XVII ${ }^{e}$ siècle, qui gouvernaient en vertu de « l'athname » du sultan, et avec le consentement de l'empereur Habsbourg. 
Cet îlot de tolérance, où quatre confessions cohabitaient légalement depuis 1568 et où le judaïsme était plus ou moins licitement pratiqué jusqu'à 1631, a reçu le nom de Jardin des fées. C'est le titre que le romancier naturaliste Zsigmond Móricz donne, en 1922, à son œuvre ouvrant une trilogie historique ${ }^{16}$. Le roman retrace les ruses et les cruautés gratuites d'un tyran capricieux, Gábor Báthory, pour y opposer l'honnêteté et la prudence tranquille d'un homme politique d'envergure, Gábor Bethlen. Le monde ottoman apparaît sous un aspect double, attirant et menaçant à la fois ${ }^{17}$. L'extase frôlant la folie est le moteur d'une scène qui met en parallèle les façons de penser occidentale et orientale. Móricz consacre quelques phrases rapides au calme de Gábor Bethlen, qui va et vient à Istanbul en habitué, « dans cette ville où rien ne presse », sans apercevoir la saleté, ni les faîtes affaissés, les voûtes croulantes sous le poids du temps (Móricz : 391). Il assiste au conseil du divan, pour obtenir du sultan le titre d'archiduc. Son affaire est liée au souhait de son allié, le pacha Iskender, de devenir pacha de Buda.

"Au cours des conversations, silencieux et calme, dans le bouquet murmurant desquelles des vipères chuintaient, on a décidé que le pacha de Buda est un menteur et un ivrogne... autour de lui, le peuple s'est mis à boire et ainsi, les murs ne sont plus gardés, les concierges ne passent pas la nuit aux portes ». (Móricz : 392).

Le terrain ainsi préparé, on commence par raconter longuement le siège de Győr par les Hongrois. De plus en plus de critiques se glissent dans le récit dont les paroles sont comprises par Bethlen, mais dont l'opportunité lui semble difficilement décodable. Comme l'imprudence des défenseurs ottomans aboutit à la perte de la forteresse, le divan exprime son consentement avec Pálfy, le vainqueur hongrois qui a fait exécuter l'agha janissaire en punition pour sa négligence. Cette prise de position (dans une autre affaire) signifie en leur langage politique que la cause d'Iskender tourne en sa défaveur. Le tour de Bethlen arrive, il doit faire un rapport de la situation en Transylvanie, en langue turque, dans le plus imagé des styles :

"Il devait s'arrêter après chaque énonciation, pour que l'interprète puisse donner une forme poétique à ses pensées de giaour ; mais il a reçu l'interprète le plus distingué, le meilleur poète qui a pomponné chaque phrase par lui dite d'un raffinement poussé jusqu'à la sophistication ». (396).

Le sultan tourne un visage froid et lointain vers Bethlen, mais ses yeux brillent en écoutant les paroles de l'interprète. La scène devient hallucinante. Les phrases bourrées de superlatifs de Bethlen sont encore multipliées en longueur et complètement transformées en divagations hyperboliques. À la fin, le sultan, dans une extase plus affolante que le vin ou l'opium, tombe par terre. Personne ne bouge ni ne s'étonne. Puis les membres du conseil prennent la parole; calmement, l'un après l'autre. Il s'agit de leur consentement et de leurs conditions (graves, d'ailleurs), prononcés d'un ton tout différent que le rapport traduit par l'interprète. Le calme froid des pachas du divan correspond parfaitement au style du protestant Bethlen, ils se comprennent. Móricz suppose donc une situation (assez bien confirmée, d'ailleurs, par l'histoire du XVII ${ }^{e}$ siècle) dans laquelle les parties commencent à se connaître et savent parler la langue de l'autre.

La tolérance de l'archiduc Bethlen semble répondre à celle de la puissance ottomane installée en Hongrie ${ }^{18}$. Selon l'opinion des chercheurs d'aujourd'hui, l'Empire ottoman n'a pas pratiqué directement les conversions, dès le moment de son installation définitive ${ }^{19}$. Les seigneurs turcs n'étaient pas pires que les compatriotes hongrois. Les habitants des zones frontalières, imposées par les deux administrations et exposées aux escarmouches continuelles vivaient, par contre, en situation vraiment difficile. La scène 
du roman de jeunesse d'István Fekete, Le Testament de l'agha de Koppány se situe très exactement dans cette zone. Le récit commence par deux duels (strictement défendus des deux côtés, d'ailleurs), l'un pour la gloire, l'autre pour l'honneur, et les deux opposent les Hongrois et les Turcs. La problématique est pourtant la question de survivre en s'unissant face à la troisième partie, au vainqueur riant, représenté par des mercenaires cruels et avides d'argent, venus des quatre coins de l'Empire Habsbourg. La solidarité des captifs, puis l'amitié des jeunes guerriers vaillants, ensuite le soutien mutuel des hommes de parole fondent enfin des relations durables, voire familiales, entre les anciens ennemis. Ce roman de Fekete, écrit en 1937 est une lecture faisant l'objet d'une option obligatoire dans les écoles primaires ${ }^{20}$.

Le début du $\mathrm{XX}^{\mathrm{e}}$ siècle ajoute également à l'image divisée des éléments de la mémoire populaire. La nouvelliste István Tömörkény se rappelle des pèlerins de Gül Baba qui, dit-elle,

« ont cheminé sur cette longue route encore dans mon enfance, en grand turban, dans leur kaftan usé, en culotte large. Il est possible qu'ils voyagent encore, mais en train déjà. Vers la fin des années 1870, les vieux Turcs faisaient encore la longue route à pied. Parfois, ils devaient monter sur un char qui passait par là. L'auberge du village les hébergeait gratis, ces gens n'avaient pas d'exigences: un morceau de pain et quelques concombres, on leur donnait cela par amitié. Arrivés dans une ville où il y avait un café, ils se reposaient pendant plusieurs jours, pour se remettre à l'aide de cette boisson familière. Ils se retiraient modestement dans un coin, ou parmi les laquais ou les hussards qui attendaient messieurs le vicomte ou le juge du casino pour les accompagner avec la lampe à la maison ».

La nouvelle (1916) raconte qu'un jeune homme qu'on connait joueur, buveur, fier de se déplacer à cheval cherche noise à un vieux pèlerin. L'autre hôte de l'auberge perdue, un vieux bouvier, essaie de ramener le jeune homme à la raison, en lui disant: Nono! (Tiens-tiens!) L'appel n'ayant pas le résultat voulu, le vieux bouvier ferme la porte ouverte. Car l'honneur (la virtù) des anciens ne permet pas de jeter dehors quelqu'un par une porte ouverte : exploit trop facile. La chose effectuée à la main, le bouvier se présente au pèlerin: "Je m'appelle Mihály Kara Török le Vieux. Ça s'écrit même comme-ça.»

Le cadre explique l'intervention et la présentation curieuse du bouvier :

«[...] chez nous ici, la longue domination turque n'a rien laissé en pierre. Seules les boulangeries à l'ancienne, les shimindjias, quelques parcelles tortueuses de ruelles, un passage à coincer des chiens, où deux chariots ne passeraient pas. Telle maison, sans porte donnant sur la rue, et qui est accessible depuis le voisin, une autre, affaissée, basse, sans fenêtre sur la ruelle. Rien d'autre. Ni mosquée, ni djami, même pas sur les anciennes cartes. En revanche, beaucoup de gens nommés Török sont restés bien vivants, et dans les hameaux, les familles appelées Kara, et conséquemment à leur nom (Kara veut dire noir), malgré les temps écoulés, ces gens ont pour la plupart la peau foncée, les cheveux noirs et autrefois, ils portaient la barbe [...] ».

Selon Tömörkény, la mémoire réside donc dans les hommes, et non pas dans les objets, surtout lorsque l'on connaît leur rareté dans la plaine hongroise.

«Les pèlerins se sont déplacés en silence, paisiblement, dans le café, ils fumaient la petite pipe sans parler, et si, enfant curieux, je les côtoyais, leur main s'est reposée un moment sur la tête d'enfant. [...]

"Les pèlerins se déplaçaient en silence, paisiblement, dans le café, ils fumaient la petite pipe sans parler, et si, enfant curieux, je les côtoyais, leur main se reposait un moment sur la tête d'enfant. [...]

Il y avait parmi eux des connaissances qui savaient un peu parler hongrois, car ils faisaient l'aller-retour chaque année. On a dit de ces gens qu'ils faisaient le pèlerinage au nom 
d'autres, non pour eux-mêmes, une fondation pieuse de riches Turcs leur payait les frais $d u$ voyage pour que la tombe du saint qui reposait dans le château de Buda, turc jadis, ne sombre pas dans l'oubli ».

nouvelle est une manifestation éclatante de la métamorphose considérable de l'image de l'Empire ottoman au cours des siècles en Hongrie. Au quinzième siècle, un des premiers témoignages européens présente un régime bien ordonné, dont les valeurs morales constituent un exemple à suivre par la chrétienté même. Vers le milieu du XVI ${ }^{e}$ siècle, Hans Dernschwam ne trouve dans l'Empire que des «bizarreries ridicules et vicieuses ». Au cours de la même période, les chansons de geste de Tinódi montrent un sultan Soliman ennemi d'envergure des puissances occidentales ; un siècle plus tard, le poète Miklós Zrínyi sublime ce monarque en une figure légendaire. Zrínyi, lui-même chef militaire qui se bat avec succès contre le Turc, chante son ancêtre comme le premier «athleta Christi » dans son épopée Le péril de Sziget en mettant en valeur sa vaillance par la reconnaissance de l'héroïsme de preux Ottomans. Le XIX siècle ouvre deux nouvelles voies : le romantisme de Jókai peint une âme profonde et orientale, des désirs et des secrets inassouvis; le réalisme de Tömörkény garde le souvenir populaire des contacts des petites gens. Le $\mathrm{XX}^{\mathrm{e}}$ siècle exploite le sujet ottoman tout en hésitant entre l'image de l'ennemi séculaire - dans la littérature soutenant les fiertés nationales - ; et une autre tendance qui présente l'image d'une cohabitation paisible permettant aux deux peuples de s'épauler dans le quotidien.

\section{BIBLIOGRAPHIE}

Dernschwam, Hans, (1984), Törökországi útinapló, Lajos Tardy, éd., Erdély, Besztercebánya, Törökországi útinapló, Budapest : Európa.

Dobrovits , Mihály, Sándor Őze, (2001), «Pázmány Korán-cáfolatának elõzményei : A török XVI. századi magyarországi megítélése» [Les antécédents de la réfutation du Coran par Pázmány: l'opinion sur le Turc en Hongrie au $16^{\mathrm{e}} \mathrm{s}$.], Emil Hargittay, éd., Pázmány Péter és kora, Piliscsaba : PPKE BTK, pp. 62-70.

Georgius de Hungaria, [Anonyme de Sebes/Scepusius/Mühlbach], Értekezés a törökök szokásairól, viszonyairól és gonoszságáról [Tractatus de Moribus Condicionibus et Nequicia Turcorum], Lajos Tardy, éd., Rabok, követek, kalmárok az oszmán birodalomról, Budapest : Gondolat, (1977), pp. 49-153.

Jókai, Mór, Szolimán álma, HUNGARIAN ELECTRONIC LIBRARY: http://www.mek.iif.hu

Kálmány, Lajos, (1952), Történeti énekek és katonadalok [Chants historiques et militaires], Budapest : Magyar Néprajzi Társaság, p.143.

Móricz, Zsigmond, (s.d), Tündérkert, Budapest : Athenaeum.

Rúzsás, Lajos, (1986), «A magyar közvélemény útkeresése Mohács után a XVI. században», Lajos Rúzsás, Ferenc Szakály, éds, Mohács-tanulmányok, Budapest : Akadémiai.

Cahiers balkaniques, 36-37| 2008 
SZEPESI, György, (1999), Sásvár bég históriája, Pál Ács, éd., Régi Magyar Költők Tára XVI. sz., vol. XI, Akadémiai-Orex Kiadó, Budapest, pp. 157-172 ; 429-443.

TiNóDI, Lantos Sebestyén, Cronica, HUNGARIAN ELECTRONIC LIBRARY: http://www.mek.iif.hu

TÖMÖRKÉNY, István, (1977), «Gül Baba zarándokai», Válogatott novellái, Budapest, Szépirodalmi, pp. 857-860.

VÁLASZÚTI, György, (1981), Pécsi disputa, Katalin Németh S., éd., RMPE 5, Budapest : Akadémiai.

VARJAS, Béla, éd., (1987), Irodalom és ideológia a 16-17. században, Budapest : Akadémiai.

\section{NOTES}

1. Les relations et les chartes hongroises ne sont pas les seules à confondre des tribus, des ethnies et des peuples différents ; pourtant, vers le milieu du XIV ${ }^{\mathrm{e}}$ siècle, les contacts directs hungaroottomans se sont multipliés. Après 1387 , durant le règne de Sigismond de Luxembourg, roi de Hongrie, plus tard empereur, les échauffourées frontalières sont devenues régulières et les contacts quasi permanents. La bataille de Kosovo Polje (Rigómezö) en 1389, puis celle de Nicopolis en 1396 ont donné une image de plus en plus distincte d'une menace militaire et d'une force organisatrice indomptable.

2. En premier, le bavarois Hans Schiltberger, captif de la bataille de Nicopolis, écrit une sorte d'itinéraire qui survole les territoires dominés par l'Empire ottoman, en suivant la cour de Bayazid $\mathrm{I}^{\mathrm{er}}$ et de Timour-Lang.

3. Georgius de Hungaria, saxon de Transylvanie, connu dans l'historiographie hongroise pendant longtemps comme l'Anonyme de Sebes, décrit la vie et les habitudes de diverses couches sociales dans lesquelles il s'est intégré. Élève de l'école de Sebes, il est tombé en esclavage lors de la prise de la fortification, en 1438. Libéré après vingt ans de captivité, il devient dominicain et écrira son œuvre sur les mœurs et les conditions de la Turquie dans les années 1470. La vivacité et le ton personnel de son traité sont dus au fait que lui a pu garder et emmener ses notes.

4. Saxon de Bohême, originaire de la bourgeoisie aisée, fréquente les universités de Vienne et de Leipzig, ainsi que les cercles curials les plus distingués. Il s'inscrit à l'université de Vienne à l'âge de 14 ans, en 1507. Son appartenance à la Natio Hungarica, comme c'est le cas encore tout au long $\mathrm{du} \mathrm{XVI} \mathrm{I}^{\mathrm{e}}$ siècle, ne donne pas d'indication concernant son ethnie et sa langue maternelle. Reçu bachelier à l'université de Lipsia en 1510, il est rentré en Hongrie et s'est installé à Buda, où les humanistes tchèques et moraviens formaient un cercle autour de la cour du chancelier Stanislas Thurzó et du cardinal Tamás Bakócz, primat de Hongrie. Dernschwam a participé au 5e concile de Latran, puis s'est engagé auprès du tuteur du jeune Louis II, Jérôme Balbi. En 1515, il a assisté à la rencontre entre l'empereur Maximilien et Ladislas de Jagellon au cours de laquelle furent arrangés leurs liens dynastiques par le mariage double entre Louis II et Marie d'Autriche (future Marie de Hongrie, gouvernante de Belgique) et Ferdinand d'Autriche et Anne de Jagellon. À partir de 1517, nous retrouvons le jeune humaniste au service de la firme de la famille Thurzó, à Besztercebánya. Il devient, par la suite, le confident et le commis plénipotentiaire des Fugger. Après la défaite de Mohács et la mort du jeune roi, sa femme apostrophée la "veuve chrétienne » par Érasme, Marie de Hongrie, a conservé certains fiefs dont les mines d'or et de cuivre des Hauts Pays. Ses vagues tentatives d'organiser une défense contre une éventuelle attaque ottomane sont jugées ridicules par Dernschwam, alors « factor» (commissaire) de Beszterce. Les rapports entre les Fugger, exploitant les mines et la puissance impériale, sont devenus de plus en plus encombrants et malaisés ; en 1547, Antoine Fugger se rappelle avec nostalgie des affaires basées sur l'honneur, passées avec les seigneurs hongrois de la cour de Szapolyai, roi rival de Ferdinand, entouré d'une bureaucratie, contrariante et de mauvaise foi. 
5. György Szepesi accuse aussi le bey Sasvár dans un chant historique. Le bey est constamment en agitation pour s'emparer d'un pouvoir de plus en plus grand, et ce n'est pas pour rendre service au sultan ou pour la gloire d'ajouter un joyau au turban de l'empereur qu'il massacre les Hongrois, mais c'est uniquement la haine qui l'y pousse (158).

6. Un siècle plus tard, le comte Zrínyi fait grief au bey Arslan de s'enivrer d'opium et de lancer des provocations inutiles, mettant ainsi sa vie en danger. Résultat moral: Arslan s'enfuit lâchement devant la riposte (Le siège de Sziget, $2^{\mathrm{e}}$ chant).

7. Dernschwam : 449. Boussourman (büssürmen) - musulman, mais, dans l'usage de Dernschwam, e. chrétien converti à l'Islam.

8. Les routes empruntées par les ambassades ont suivi, en gros, les chemins battus, depuis les légions romaines, les frontières des deux empires restant flexibles selon les campagnes, les points de passage se sont tout de même vite établis et restaient constants.

9. Az tereknek hiti erőssen aszt tartja, / Ha hittel, szép szóval kavort megcsalhattya, / Ajándokkal, vagy lopva elragadhattya, / Isten azért néki mennyországot adgya. (Tinódi: [XVII.] Príni Péternek Majlát Istvánnak és Terek Bálintnak fogságokról)

10. La guerre commencée, Tinódi fait une remarque à propos du niveau de la technique militaire de l'armée du pacha Kazul : ses gens tombent sous le feu des fusils dont ils n'ont pas peur, parce qu'ils ne savent pas tirer. (XIV.85-89).

11. Le troisième tiers du chant dessine la douleur des proches de Bálint Török restés sans chef de famille. Encore un cas triste, celui de Lajos Pekri qui montre que «l'empereur turc ne tient pas sa parole ».

12. Ali a vaincu le capitaine général de l'armée royale, Erasmus Teufel dans une bataille régulière à Palást, première rencontre ouverte depuis longtemps. Si Ali réussit à prendre huit châteaux durant la campagne de 1552, c'est grâce aux hostilités internes qui opposent les défenseurs hongrois et les mercenaires impériaux.

13. Tinódi insiste sur le fait que les troupes turques qui ont suivi Vas, l'ont « nettoyé » de tous ses biens, c'est-à-dire, l'ont privé de son argent et même de son cheval.

14. Il s'agit d'une autre histoire lorsqu'il fut obligé de fuir jusqu'en Turquie, chassé par ses coreligionnaires comme traître et lâche.

15. L'historien Szamosközy rétorque avec une ironie mordante l'accusation d'avoir passé un pacte honteux avec l'Empire ottoman: «Ces dernières années, nous avons bien appris ce qu'on peut espérer de la nation allemande ", dit-il.

16. Móricz a édité la trilogie Transylvanie en 1933.

17. Une scène est particulièrement poétique, même si les images évoquent au même titre l'exubérance ciselée de l'art nouveau et les arabesques du XVII siècle. Le jeune archiduc, Báthory, fait parler son ambassadeur du Coran, car « Ce temps-là, le goût turquisant chatouilla, comme un poison doux, la curiosité de la couche noble des chrétiens, surtout des protestants. La Réforme a éclairé les esprits : l'homme est libre d'examiner toutes les choses du monde [...] Le pouvoir extraordinaire du Turc, sa force, piétinant sur tout et dominant tout, fit impression, et le contact personnel avec le Turc donna un avant-goût de la joie, des délices et de la secrète volupté orientaux.» (316-17) Suit une description du Paradis donné par l'ambassadeur qui séduit le public ; les favoris et les favorites du jeune tyran suivent le conte avec avidité : «Ils ont écouté dans un vertige les jets de cent mille ans et les femmes, comme chatouillées, se sont mises à rire. Géczy, tout comme s'il était devenu Turc lui-même, continua à expliquer l'Alcoran avec une piété quasi turque. «Il y a soixante-dix mille châteaux en rubis sur chaque champ, chaque château a soixante-dix mille cours en hyacinthe, il y a soixante-dix mille maisons faites en or dans chaque cour, [...] dans chaque palais, il y a soixante-dix mille trônes d'or et sur chaque trône, il y a soixante-dix mille lits faits en pourpre et en argent brillant... » Les femmes s'écrièrent, émues des nombres monstrueux, mais Géczy, grave et sérieux, continua : «Et autour de chaque lit, il y a soixante-dix mille fillettes faites en lueur paradisiaque. » Elles se blottirent contre la poitrine 
l'une des autres, le merveilleux leur chatouilla le cœur, la volupté leur donna la chair de poule. » (317) Le texte de l'ambassadeur est un amas de mots jugés phonétiquement purs et beaux dans les jeux linguistiques exercés dans les cafés littéraires à l'époque de Móricz et, par ce fait même, un pastiche naturaliste du style baroque, modérément archaïsant. Le rythme de la prose s'accélère, et quand il arrive, essoufflé, à la parole de Dieu ordonnant à l'archange Gabriel d'enlever le voile devant la vision face à face, la voix de l'ambassadeur meurt et la soirée se métamorphose en une orgie spirituelle, d'autant plus chère que la turcophilie est prohibée. La soif de connaître les pensées et la religiosité de l'ennemi est une idée du XX ${ }^{\mathrm{e}}$ siècle, quand, devant les lecteurs de Móricz, il est notoire que les bannis de l'empire Habsbourg ont trouvé refuge pendant des siècles chez l'ancien ennemi.

18. Plusieurs cas prouvent que les autorités ottomanes, dès la deuxième moitié du XVII ${ }^{\mathrm{e}}$ siècle, protègent les confessions protestantes contre la poursuite des catholiques. Il semble que le protestantisme était en position favorisée sur les terres ottomanes, mais il arrivait également que les autorités catholiques et musulmanes jugent unanimement (quoique séparément) une affaire morale ou judiciaire. La dispute de Nagyharsány en 1574 a mal tourné pour les représentants antitrinitaires, tués au lieu d'être convertis ; par la suite, ce cas de meurtre était puni respectivement par l'évêque de Pécs et par le pacha de Buda, du point de vue ecclésiastique d'une part, et de la puissance temporelle de l'autre. Les autorités ottomanes étaient souvent saisies en cas de litiges entre Hongrois également. Le cas de la Dispute de Pécs en 1588 n'est pas unique, avec le prêtre catholique et le bey du château regardant paisiblement la discussion théologique d'un prédicateur antitrinitaire et d'un représentant de la confession helvétique.

19. Les moyens indirects, en revanche, comme l'imposition différenciée, le mélange conscient des ethnies et des confessions, en un mot, les techniques de division ont été largement utilisées. L'étude de ces techniques a été nuancée ces vingt dernières années par les historiens hongrois. Selon le témoignage du folklore, le devchirme (la levée d'enfants), était ressenti comme l'impôt le plus cruel. Dans un conte recueilli par Lajos Kálmány en 1916, le fredonnement d'une nourrice, énumérant les signes du corps de son fils, jadis pris en devchirme, révèle sa véritable identité devant le chef de famille qui écoute dans la pièce à côté, et une scène de reconnaissance clôt l'histoire en bonheur.

20. La version filmée n'égale pas les valeurs cinématographiques du film de Várkonyi, Les étoiles d'Eger tiré du roman de Géza Gárdonyi, mais jouit d'une grande popularité parmi les films « turcs».

\section{RÉSUMÉS}

Dans la culture hongroise, 150 ans de proche cohabitation avec l'Empire ottoman ont laissé d'innombrables traces. En littérature, la richesse relative en documents ne nous permet que de frôler la «question turque ». Nous proposons ici une récurrence fondamentale, l'honneur et tout particulièrement la parole donnée. Cette problématique peut être envisagée selon trois axes. Les engagements personnels - politiques et militaires - ; la question des conversions, recoupant le domaine de la religion; le fonctionnement de la cohabitation, plus complexe. Les textes illustrant ces trois approches sont d'une grande diversité. Les mémoires d'esclave ou les chants épiques y tiennent une place égale avec le roman du XIX ${ }^{\mathrm{e}}$ siècle.

L'image de l'Empire ottoman a subi une métamorphose considérable au cours des siècles en Hongrie. Au quinzième siècle, un des premiers témoignages européens présente un régime bien 
ordonné, dont les valeurs morales constituent un exemple à suivre pour la chrétienté. Vers le milieu du XVI ${ }^{\mathrm{e}}$ siècle, Hans Dernschwam ne trouve dans l'Empire que des «bizarreries ridicules et vicieuses ». Au même moment, les chansons de geste de Tinódi montrent le sultan Soliman en ennemi redouté des puissances occidentales. Un siècle plus tard, le poète Miklós Zrínyi sublime ce monarque en figure légendaire. Le XIX ${ }^{\mathrm{e}}$ siècle ouvre deux nouvelles voies : le romantisme de Jókai peint une âme profonde et orientale; le réalisme de Tömörkény s'intéresse aux petites gens. Le XX ${ }^{\mathrm{e}}$ siècle exploite le sujet ottoman tout en hésitant entre l'image de l'ennemi séculaire, et celle d'une cohabitation paisible des deux peuples.

The 150 years of the close cohabitation with the Ottoman Empire has certainly left numerous traces in the Hungarian culture. With regard to literature, the relative poorness of available documents does not allow us to even to venture in the road of the "Turkish question". Here we shall study what it appears to be a subject that is fundamental as well as recurring, that of the question of honor and the word given. We shall further analyze these questions this along three axes: personal engagement - political and military - religious conversion, where we find al sorts of topics relating to religion and finally coexistence, which is more complex by itself. The texts illustrating those three different approaches are of a great diversity. Within such corpus we may find slave memoirs, epical songwriting, as well as the equally important XIX ${ }^{\text {th }}$ century novels.

The image of the Ottoman Empire has been through a considerable transformation over time in Hungary. In the fifteenth century one of the first existing European accounts of the country shows a well regulated regime, with moral values that constitute an example the whole Christian world should probably follow. Around the middle of the sixteenth century though, Hans Dernschwam finds that the empire is full of "ridiculous and vicious eccentricities". At the same time the epic poems of Tinódi show that sultan Suleiman was an enemy feared by the western forces. A century later, the poet Miklós Zrínyi makes a legend out of the same monarch. New voices appear during the XIXth century: Jókai's romanticism depicts the deep oriental soul, while Tömörkény's realism portraits the little people in particular. In the $\mathrm{XX}^{\text {th }}$ century, the ottoman element may be represented either as the incarnation of an age-long enemy, or as the people Hungarians are peacefully coexisting.

\section{INDEX}

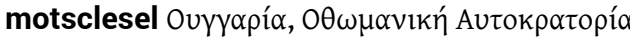

Thèmes : Littérature

Index géographique : Hongrie

motsclesmk ОТОМАНСКАТА ИМПЕРИЈА, УНГАРИЈА

motsclestr Macaristan, Osmanlı İmparatorluğu

Mots-clés : Dernschwan Hans , Chalcondylis Laonikos (1423-1490), Georgius de Hungaria

(1422-1502), Jokai Mór (1825-1904), Zrínyi Miklós (1620-1664), Tömörkény István (1866-1917),

Dernschwan Hans (XVIe siècle), Tinódi Sebestyén (1510-1556), Tinódi Sebestyén (1510-1556),

Zrínyi Miklós (1620-1664)

Index chronologique : dix-neuvième siècle, Empire ottoman, seizième siècle, vingtième siècle, dix-septième siècle

Keywords : hungarian litterature, ottoman, Zrinyi Miklos (1620-1664), Jokai Mór (1825-1904), Tinódi Sebestyén (1510-1556), Hungary, nineteenth century, sixteenth century, seventeenth century, thirteenth century, Literature 